\title{
The boundaries of mental health first aid
}

$\mathrm{S}$ ometimes it just takes a willingness to ask tough questions: "Are you suicidal? How would you kill yourself? Do you have the means? Have you attempted suicide in the past?"

In other cases, the preferred protocol is to contact emergency services, while trying to reduce the level of distress, as in the case of dealing with someone who appears to be suffering from a panic attack, or who is in the throes of a psychotic episode.

Such are the nuances of mental health first aid, as presented in a twoday course offered by Mental Health First Aid Canada to the general public. While its content may be altogether familiar to physicians, it is certainly an educational and often eye-opening experience for the average joe, if only because, as with physical first aid or cardiopulmonary resuscitation, it seems that the nature of the assistance provided is highly tailored to the nature of the disorder. As such, it could be a useful tool that physicians can point toward for people looking for guidance on how to deal with family or friends who appear to be suffering mental health problems.

Who knew, for example, that when talking to someone with schizophrenia that it was important to find a way to cut through the potential clutter of internal voices? To that end, people taking the first aid course are asked to simulate what it is like to carry on a conversation with someone who has voices in his head, spouting phrases such as "don't trust him" or "he's a spy." So while pairs of students in the first aid class are asked to babble with one another, a third is asked to roll up a piece of paper and speak calmly, slowly and concisely into the ear of one of the students, repeating reassurances as often as is necessary.

And who knew that it is actually advisable to call the police if someone is suicidal because they have the authority to take that person to a physician for

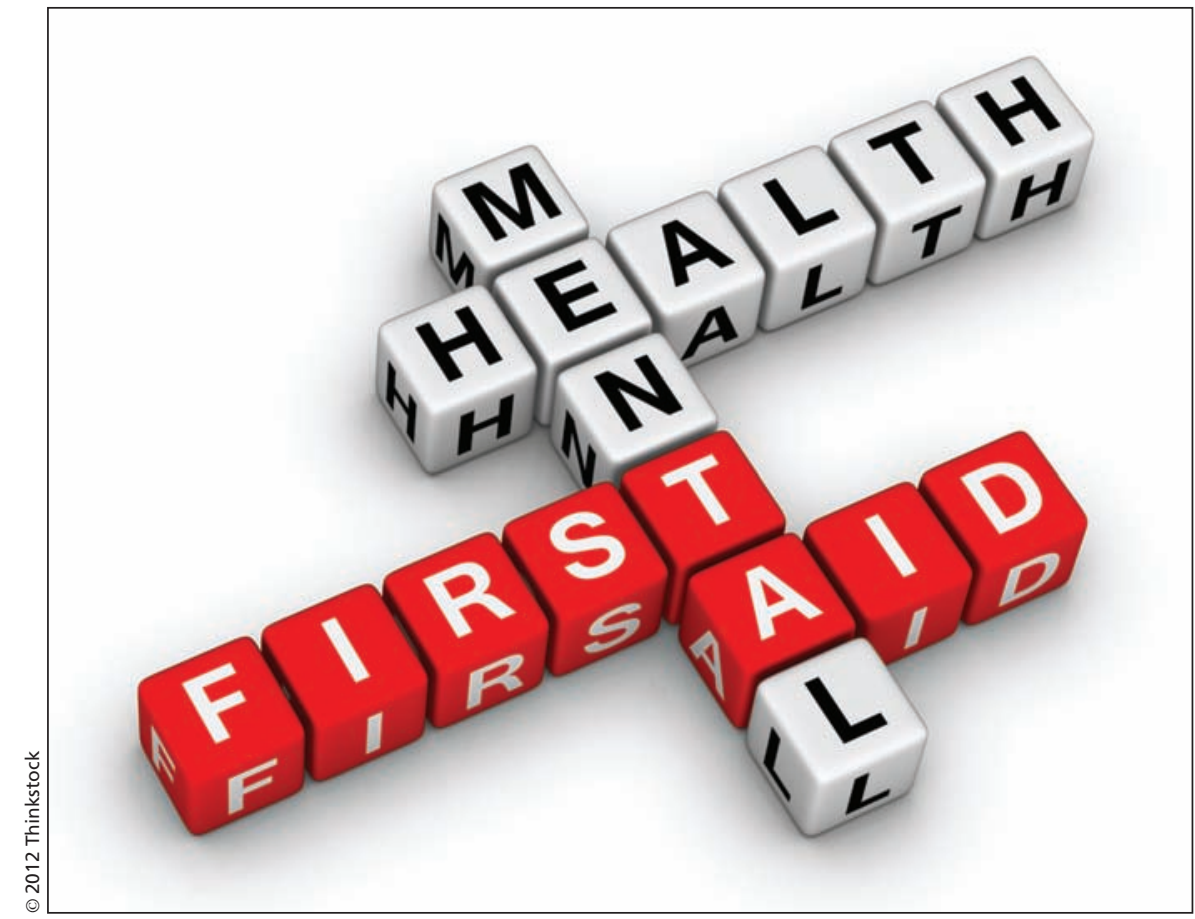

Despite the prevalence of mental health problems, many people don't recognize, or misinterpret, the symptoms of a developing condition.

evaluation? Or to press them to talk about their suicide plans?

It's a common misconception that asking someone if they've thought about killing themselves will drive them to take action, says Christine Slepanki, manager of the youth psychiatry program at the Royal Ottawa Mental Health Centre in Ontario and one of the Mental Health First Aid instructors.

On the contrary, a willingness to talk about intent makes it apparent that someone cares and often just being asked and given the opportunity to express bottled-up emotions can be a relief to a person who's contemplating suicide, Slepanki adds. "We're talking life or death," she tells the class. "There are no boundaries."

The course outlines various mood, anxiety, psychotic and substance-abuse disorders, while trying to teach participants a proper response to mental health emergencies. The aim isn't to make counsellors or therapists of participants but rather, to teach members of the gen- eral public how to intervene in a mental health crisis, even in cases where the troubled person is unaware or in denial of the problem. The ultimate aim is to guide people toward treatment, and to provide support when professional help is not immediately available.

But another objective of the program is to reduce the stigma of mental health problems. "A lot of people would say the stigma is as bad as the illness," says Hilary Jocelyn, a social worker on the centre's psychiatric outreach team and another instructor. "So if we run a course about mental health, we have to tackle that."

One in five Canadians experiences a mental health problem annually, and one in three over the course of a lifetime, according to the Public Health Agency of Canada (www.phac-aspc .gc.ca/publicat/human-humain06/pdf /human_face_e.pdf). Nearly 3900 Canadians committed suicide in 2009 (www .statcan.gc.ca/tables-tableaux/sum-som /101/cst01/hlth66a-eng.htm). 
Those numbers speak to the need for the mental health first aid program, says Denise Waligora, manager of program education at the Mental Health Commission of Canada.

Despite the prevalence of mental health problems, many people don't recognize, or misinterpret, the symptoms of a developing condition and are unaccustomed to offering support to family, friends, colleagues, or strangers, she says. "People don't want to get involved, people are too rushed in their lives and people don't understand mental health. That's what's so important about the course; it makes it so people are okay and comfortable talking about it."

"The support that people have is one of the most important things in the process of recovery," Waligora adds. "So the more Canadians that we can train to be mental health first-aiders, how much more supportive communities we'll have."
Nearly 59000 Canadians have completed the course and almost 700 have been trained as instructors, Waligora says. Although first created in 2001 in Australia and now offered in 18 countries, it didn't take hold within Canada until 2006, when Alberta and Manitoba made it available. The Mental Health Commission of Canada took control of the program in mid-2010 and participation has since soared, she adds.

As with physical first aid, which teaches a response based on what are known as the ABCs (for airway, breathing and circulation), mental health first aid has its own response-based acronym and mnemonic, known as ALGEE, which stands for:

- Assess the risk of suicide and/or harm, calling emergency medical services or encouraging the person to contact a local crisis team if the risk is deemed severe;

- Listen non-judgmentally;
- Give reassurance and information, for example, by saying that the problem does not indicate weakness of character or, in the case of a disorder like depression, that it is common and treatable;

- Encourage the person to seek appropriate professional help; and

- Encourage the person to seek other supports, such as reaching out to family and friends or exploring selfhelp strategies.

Along with the basic mental health first aid course, there is an expanded version for adults who deal with youth, including additional modules on selfharm and eating disorders. The commission is now developing a course tailored to Aboriginal peoples, as well as a geriatrics course that will include information on mental health problems such as delirium and dementia. - Michael Monette, CMAJ

CMAJ 2012. DOI:10.1503/cmaj.109-4298 